\title{
LA EVALUACIÓN FORMATIVA COMO ELEMENTO PARA VISIBILIZAR EL DESARROLLO DE COMPETENCIAS EN CIENCIA Y TECNOLOGÍA Y PENSAMIENTO CRÍTICO
}

\author{
Formative assessment as an element to visible the development of competences in \\ science and technology and critical thinking.
}

A avaliação formativa como elemento para visibilizar o desenvolvimento de competências em ciência e tecnologia e pensamento crítico

\section{Vanessa Ortega-Quevedo (1) \\ Cristina Gil Puente (2)}

(1) Universidad de Valladolid. Teléfono: 921112300. Correo electrónico: vanessaortegaquevedo@gmail.com

(2) Universidad de Valladolid. Teléfono: 921112300. Correo electrónico: cgil@dce.uva.es

\section{Resumen}

Este trabajo presenta una experiencia cuyas finalidades principales consisten en mejorar las competencias en ciencia y tecnología y desarrollar Pensamiento Crítico de los alumnos de Educación Primaria, mediante la implementación de una secuencia didáctica que toma como base la evaluación formativa y el diálogo. Dicha propuesta se ha llevado a cabo en el área de Ciencias Naturales, concretamente en cuatro centros educativos de Segovia en $6^{\circ}$ curso de Educación Primaria. Los resultados obtenidos muestran que los estudiantes han mantenido una participación activa en el proceso de evaluación que les ha permitido reconstruir sus aprendizajes a partir de las intervenciones realizadas por sus compañeros o por el propio maestro. En conclusión, a pesar de las dificultades encontradas la experiencia ha resultado positiva y refleja que aplicar la evaluación formativa permite visibilizar el desarrollo de las concepciones sobre Naturaleza de la Ciencia y la Tecnología y las capacidades de Pensamiento Crítico.

Palabras clave: Evaluación formativa; pensamiento crítico; naturaleza de la ciencia y la tecnología; educación primaria 


\begin{abstract}
This project presented an experience whose aim is improving skills in science and technology and the development of Critical Thinking, in Elementary Education. This is achieved through the implementation of a didactic sequence based on formative assessment and dialogue. This proposal has been put into practice in the area of Natural Sciences, specifically in four educational schools in Segovia (Spain) in 6th grade Elementary Education. The results show that the students have had an active participation in the assessment process that has allowed them to reconstruct their learning from the results made by their classmates or by the teacher. To conclude, the proposal has been positive and reflects that to apply the formative assessment allows to visualize the development of conceptions about the Nature of Science and Technology and the capacities of Critical Thinking.
\end{abstract}

Keywords: Formative assessment; critical thinking; nature of science and technology; elementary education

\title{
Resumo
}

Este trabalho apresenta uma experiência cujos principais objetivos são melhorar as habilidades em ciência e tecnologia e desenvolver o Pensamento Crítico de alunos do Ensino Fundamental, através da implementação de uma sequência didática baseada em avaliação formativa e diálogo. Esta proposta foi realizada na área de Ciências Naturais, especificamente em quatro centros educacionais de Segóvia no $6^{\circ}$ ano. Os resultados obtidos mostram que os alunos mantiveram uma participação ativa no processo de avaliação que lhes permitiu reconstruir sua aprendizagem a partir das intervenções realizadas pelos colegas ou pelo professor. Em conclusão, apesar das dificuldades encontradas, a experiência tem sido positiva e reflete que a aplicação da avaliação formativa permite visualizar o desenvolvimento de concepções sobre a Natureza da Ciência e Tecnologia e as capacidades do Pensamento Crítico.

Palavras-chave: Avaliação formativa; pensamento crítico; natureza da ciência e tecnologia; ensino primário 


\section{Introducción}

Las características de la sociedad actual requieren el desarrollo de competencias como las de Pensamiento Crítico (PC) y las científico-tecnológicas. En esta experiencia se entiende PC como un conjunto de capacidades y disposiciones semejantes a las propias del pensamiento científico de modo que estos constructos son fácilmente tratables en conjunto (Manassero \& Vázquez, 2017; Ortega-Quevedo \& Gil 2019). Sin embargo, dichas capacidades son abstractas, de modo que se pretende visibilizar su desarrollo mediante la evaluación formativa.

La evaluación formativa se entiende como el proceso en el cual docentes y estudiantes mejoran gracias a las valoraciones que se producen durante el proceso de enseñanzaaprendizaje (Popham, 2013; López, 2017). Esta forma de entender la evaluación permite evaluar la significatividad de los aprendizajes del alumnado, en relación con el desarrollo de su competencia científica (Pedrinaci, 2012) y de PC. La propuesta de evaluación se integra de un modo mimético en el proceso de enseñanza-aprendizaje, de forma que la evaluación es utilizada "como medio de perfeccionamiento sobre la marcha" (Rosales, 1988, p.107).

El modelo de evaluación formativa empleado, por las características de la experiencia es el comunicativo-crítico. Por tanto, se implementa una evaluación basada en el diálogo y centrada en el desarrollo de competencias (López, 2017). Durante este proceso, la acción evaluadora consiste en la conducción de argumentaciones hacia lo que los científicos establecen como concepciones aceptables sobre Ciencia, Tecnología y Sociedad (Acevedo \& García; 2016, \& Vázquez, Acevedo \& Manassero, 2004).

\section{Contextualización}

La experiencia didáctica se ha implementado en cuatro centros educativos públicos de Educación Primaria de Segovia. La distribución de los participantes en los distintos centros queda reflejada en la Tabla 1.

Tabla 1.

Contexto de implementación

\begin{tabular}{ll}
\hline Centro & Total estudiantes \\
\hline 1 & 43 \\
2 & 36 \\
3 & 19 \\
4 & 19 \\
\hline
\end{tabular}

La evaluación formativa como elemento para visibilizar el desarrollo de competencias en ciencia y 


\section{Diseño y desarrollo}

La experiencia didáctica diseñada se divide en tres sesiones de una hora y está orientada a desarrollar competencias científico-tecnológicas basadas en aspectos no epistémicos de la Naturaleza de la Ciencia y la Tecnología (NdCyT), así como capacidades de PC. El tratamiento de los temas de $\mathrm{NdCyT}$ y PC se realiza de forma explícita de una forma trasversal a través de la enseñanza de contenidos del currículo de Educación Primaria (bloque 4, Materia y Energía). Este tratamiento se introduce en el uso de la terminología adecuada, el planteamiento de ítems y actividades que permiten enfatizar los factores relacionados con estas competencias y mediante preguntas de inducción a la reflexión.

Cada sesión se divide en tres partes: A) activación de conocimientos previos y procesos de PC (rutinas de pensamiento); B) diálogo de presentación de contenidos curriculares; C) consolidación mediante la reflexión inducida a partir de preguntas controvertidas y su debate. El tratamiento de contenidos tanto curriculares como de NdCyT y PC se recoge en la Figura 1.

\begin{tabular}{|c|c|c|c|}
\hline Sesiones & A) & B) & C) \\
\hline 1 & Argumentación & $\begin{array}{l}\text { gia, propiedades y sus manife } \\
\text { Influencia de la Ciencia y la } \\
\text { Tecnologia en la Sociedad } \\
\text { (ICTS) }\end{array}$ & $\begin{array}{l}\text { taciones } \\
\text { Argumentación } \\
\text { Comprobación de hipótesis } \\
\text { ICTS }\end{array}$ \\
\hline 2 & $\begin{array}{l}\text { Fuentes de energía renova } \\
\text { Argumentación } \\
\text { Comprobación de hipótesis } \\
\text { ICTS } \\
\text { Construcción social de la } \\
\text { Tecnologia (CST) }\end{array}$ & $\begin{array}{l}\text { les y no renovales e impactos } \\
\text { la energía } \\
\text { Argumentación } \\
\text { ICTS } \\
\text { CST }\end{array}$ & $\begin{array}{l}\text { ambientales relacionados con } \\
\text { Argumentación } \\
\text { Comprobación de hipótesis } \\
\text { ICTS } \\
\text { CST }\end{array}$ \\
\hline 3 & $\begin{array}{l}\text { La fa } \\
\text { Argumentación } \\
\text { ICTS } \\
\text { CST }\end{array}$ & $\begin{array}{l}\text { turación hidráulica y sus con } \\
\text { Argumentación } \\
\text { Comprobación de hipótesis } \\
\text { ICTS } \\
\text { CST }\end{array}$ & $\begin{array}{l}\text { ecuencias } \\
\text { Argumentación } \\
\text { Comprobación de hipótesis } \\
\text { ICTS } \\
\text { CST }\end{array}$ \\
\hline
\end{tabular}

Figura 1. Contenidos

\section{Evaluación}

En la Figura 2 se recogen las técnicas e instrumentos utilizados para valorar los aprendizajes. La información extraída de los distintos instrumentos de evaluación se analiza mediante ítems de observación relacionados con categorías de análisis cualitativo (Figura 3 y 4 ). 


\begin{tabular}{ll}
\hline \multicolumn{1}{c}{ Técnicas } & \multicolumn{1}{c}{ Instrumentos } \\
\hline Autoevaluación del alumnado & Ficha ${ }^{1}$ \\
Observación participante & Diario del maestro ${ }^{2}$ \\
Autoevaluación docente & Tabla de autoevaluación ${ }^{3}$ \\
\hline
\end{tabular}

Figura 2. Técnicas e instrumentos de evaluación

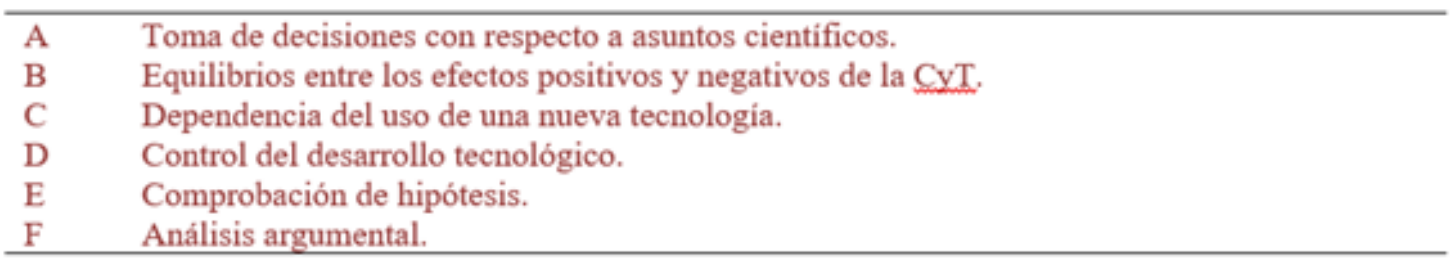

Figura 3. Categorías de Análisis Cualitativo

\begin{tabular}{lll}
\hline \multicolumn{1}{c}{ Ítems de observación } & \multicolumn{1}{c}{ CAC } \\
\hline 1 & Debate de Consolidación (DC) primera sesión & $\mathrm{A}, \mathrm{B}, \mathrm{E}, \mathrm{F}$ \\
2 & DC segunda sesión & $\mathrm{A}, \mathrm{B}, \mathrm{D}, \mathrm{E}, \mathrm{F}$ \\
3 & DC tercera sesión & $\mathrm{A}, \mathrm{B}, \mathrm{C}, \mathrm{D}, \mathrm{E}, \mathrm{F}$ \\
\hline
\end{tabular}

Figura 4. Ítems de observación

Como ejemplo se presenta el análisis del primer ítem de observación. El grupo de estudiantes con un nivel de aprendizaje más elevado tomó la iniciativa en el debate en todos los centros, exceptuando el segundo. Estos educandos, en base a las respuestas reflejadas en la ficha, orientaron el diálogo (categorías E y F) hacia las cuestiones que les suscitaban más incertidumbre (diferencias entre energía para sobrevivir y para facilitar la vida, el derroche de la energía, reflexión sobre los procesos de obtención de suministro eléctrico, etc.) (categorías A, B). En consecuencia, se trabajaron los factores deseados, permitiendo al resto de los discentes sumar sus impresiones y dudas al debate. En la Figura 5 se presenta un ejemplo de la evolución de las respuestas del alumnado extraído de las fichas de consolidación.

\begin{tabular}{lc}
\hline $\begin{array}{l}\text { Respuesta } \\
\text { inicial }\end{array}$ & $\begin{array}{c}\text { Respuesta tras la puesta en común y } \\
\text { reconstrucción de aprendizajes }\end{array}$ \\
\hline "si porque no tendriamos ni luz ni nada artificial" "la energía nos mantiene vivos, sin ella no nos \\
& $\begin{array}{l}\text { podriamos mover o respirar y nos hace la vida más } \\
\text { fácil con la luz eléctrica" }\end{array}$ \\
\end{tabular}

Figura 5. Ejemplo del ejercicio de las fichas de consolidación. Producción 1, centro 2, grupo 3.

En el centro dos, este proceso dialógico resultó más complejo para el alumnado, pues no conseguían expresar sus ideas. En consecuencia, la participación del docente fue mayor (iniciando y conduciendo los debates), lo que implica que los resultados de los procesos de argumentación y comprobación de hipótesis no alcanzasen el mismo nivel de logro que en otros centros.

La evaluación formativa como elemento para visibilizar el desarrollo de competencias en ciencia y 


\section{Conclusiones}

La implementación de la experiencia ha conseguido que la mayor parte de los educandos haya logrado reconstruir sus conocimientos consiguiendo una mejora de sus concepciones sobre los temas de NdCyT y las capacidades de PC. Se observa que la evaluación formativa es un elemento clave para visibilizar la mejora de estos factores, donde el resultado final no es tan importante como el proceso.

Se detectan mejoras en la experiencia y en el proceso de evaluación:

- Ampliar el tiempo dedicado a asentar los contenidos curriculares básicos.

- Realizar sesiones previas en las que los dicentes puedan acostumbrarse a una metodología dialógica y activa.

- Incluir tablas de observación para sistematizar el proceso de observaciónparticipante.

- Incluir rúbricas para optimizar el proceso de evaluación de los ítems y facilitar el salto a la calificación.

\section{Referencias}

Acevedo, J. A., \& García, A. (2016). «Algo antiguo, algo nuevo, algo prestado». Tendencias sobre la naturaleza de la ciencia en la educación científica. Revista Eureka sobre Enseñanza y Divulgación de las Ciencias 13(1), 3-19. Doi: $10498 / 18010$.

López, V. M. (2017). Evaluación formativa y compartida: evaluar para aprender y la implicación del alumnado en los procesos de evaluación y aprendizaje. En V. M. López y A. Pérez (coord.). Buenas prácticas docentes. Evaluación formativa y compartida en educación: experiencias de éxito en todas las etapas educativas. León: Universidad de León, Secretariado de Publicaciones.

Manassero, M. A., \& Vázquez, A. (septiembre de 2017). ¿Hay contenidos de naturaleza de la ciencia y la tecnología y pensamiento crítico en los currículos (españoles) actuales?, X Congreso Internacional sobre Investigación en Didáctica de las Ciencias. Congreso llevado a cabo en Sevilla, España.

Ortega-Quevedo, V., \& Gil, C. (2019). La Naturaleza de la Ciencia y la Tecnología. Una experiencia para desarrollar el Pensamiento Crítico. Revista Cientifica, 2(35). https://doi.org/10.14483/23448350.14095 
Pedrinaci, E. (Coord.), Caamaño, A., Cañal, P., y de Pro, A. (2012). 11 ideas clave. El desarrollo de la competencia científica. Barcelona: Graó.

Popham, W. J. (2013). Evaluación trans-formativa. El papel transformador de la evaluación formativa. Madrid: Narcea.

Rosales, C. (1988). Criterios para una Evaluación Formativa. Objetivo. Contenido. Profesor. Aprendizaje. Recursos. Madrid: Narcea.

Vázquez, A., Acevedo, J. A., \& Manassero, M. A. (2004). Consensos sobre la naturaleza de la ciencia: evidencias e implicaciones para su enseñanza. Revista Iberoamericana de Educación, 34(1), 1-36. Recuperado de: rieoei.org/deloslectores/702Vazquez.PDF 